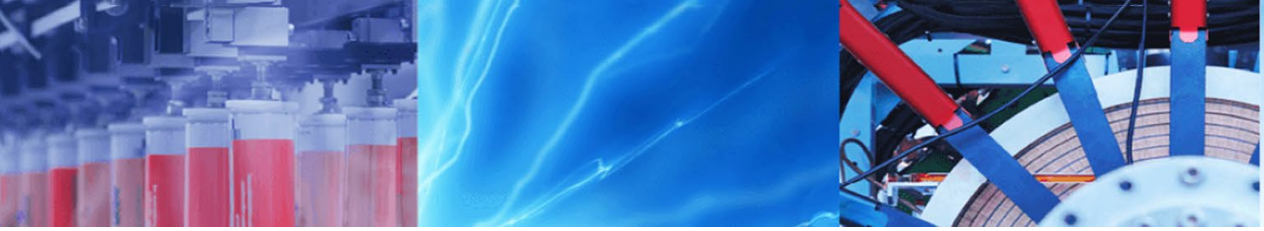

Research Article

\title{
Health and safety control measures and MR quality control results in the MRI units of two public hospitals within the Mangaung metropolitan
}

\author{
Phoka C. Rathebe ${ }^{1}$ (1)
}

Received: 8 February 2021 / Accepted: 22 June 2021

Published online: 10 July 2021

(C) The Author(s) 2021 OPEN

\begin{abstract}
This study aimed to identify risks and hazards in the magnetic resonance imaging (MRI) units, and assess the quality compliance of the scanners within two public hospitals in Mangaung. This is a follow-up study from a previously published study that measured static magnetic fields and radiofrequency magnetic fields in the MRI units included here. An observational checklist was used to identify risks and hazards which were later fed into a baseline risk assessment to classify and review existing control measures in the MRI units of hospitals A and B. The availability of MRI Health and Safety measures were benchmarked against the latest American College of Radiology (ACR) MRI safety requirements. The probability of risk occurrence and severity of hazards were assigned a score ranging from improbable (1) to very likely (5) and minimal (1) to irreversible effect (5). The weekly quality control test results obtained from both units were measured against the ACR quality control acceptable criteria. Similar risks were observed in both MRI units but the multiplication of probability and consequence in all risk categories resulted in a moderate risk-rating score of 12.3 for hospital A and 13.1 for hospital B. Lack of demarcation of four MRI safety zones, ferromagnetic detectors, 5-gauss line, and access control in both units scored above 15 and were classified as high risk. The defective air-cooling systems influenced the temperature of the scanner room, which affected the apparent diffusion coefficient (ADC) measurements performed from 1.5 T Siemens. On a 3.0 T Philips, a low contrast object detectability had 29 spokes for ACR T2, while the percent integral uniformity for image intensity uniformity was $78.2 \%$. High and moderate risks observed in both units could be reduced by the implementation of an effective health and safety programme. The ambient temperature within the scanner room should be maintained at $21^{\circ} \mathrm{C}$ to attain well-performing ADC measurements and RF subsystems should be visually inspected and maintained regularly to obtain optimal image quality.
\end{abstract}

Keywords Risk assessment · Quality control · Hazards · Risks · Magnetic resonance imaging

\section{Introduction}

The acquisition of an image from the magnetic resonance (MR) scanner is unique and mainly depends on the inherent behaviour of hydrogen protons bound to tissues and fluids to produce an image contrast [1]. The use of magnetic fields and radiofrequency (RF) coils present a variety of safety challenges that are different from other radiological imaging processes [1]. With the increasing clinical demands for MRI, there is a need for MR workers to be trained in magnetic resonance imaging (MRI) safety to protect themselves, patients, and other healthcare

Phoka C. Rathebe, prathebe@uj.ac.za | 'Department of Environmental Health, University of Johannesburg, Doornfontein Campus, Johannesburg, South Africa.

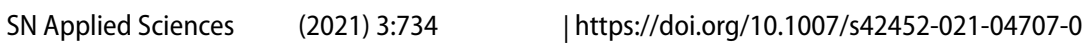


workers [2]. Despite the need for comprehensive safety training, health and safety hazards arising from main static magnetic fields (SMFs) leading to projectile force and transient effects, RF magnetic fields associated with heating and gradient magnetic fields producing acoustic noise still exist [3]. To mitigate the safety risks present in the MRI units, risk assessment is necessary to identify all hazards that could affect the health and safety of MR staff, patients, and related healthcare workers. Quality control tests could also be used to form the basis of MRI safety protocols in the MRI units. In this study, risk assessment was conducted in the 1.5 and 3 T MRI units of two public hospitals to identify all risks and hazards present in the units. Though this study focused on occupational health and safety risks, the results of quality control tests performed in both hospitals were also benchmarked with the American College of Radiology (ACR) quality control guideline document [4].

Many patients and magnetic resonance (MR) staff members encounter stray static fields when entering zone IV, creating potential health and safety accidents that could easily occur if ferromagnetic materials or implants are present near the scanner [5]. Many MRI health and safety accidents have occurred across the world in both 1.5 and 3.0 T clinical imaging, and many of those that occur in the South African MRI units remain unreported. The primary safety risks in the clinical MRI units are access control, responsibilities, and training [5]. There is also a scarcity of literature regarding hazard identification and risk assessment in the MRI units. With the increasing use of 3.0 T, MR staff should never assume MR compatibility or safe information about any device brought into the MRI units, especially if it is not documented in writing [6]. Durbridge [7] suggests that screening must be compulsory for all persons entering the MRI units, details of implantable devices must be known, and access to the area containing the $5-G$ line and the magnet room must be restricted. Since the MRI safety risks are unique and require an understanding of hardware and electromagnetic (EM) principles, the inherent risks could potentially be minimized through the development of a safety culture that relies on MR staff to develop policies that suit their facilities [1]. This is a follow-up study based on the assessment of existing health and safety control measures and clinical quality performance of the MR scanners, in the MRI units where SMFs and RF magnetic fields measurements were performed previously [8]. In the previous study [8], measured peak values for SMFs from the 3.0 T scanner at hospital A were 1300 milliTesla (mT) and $726 \mathrm{mT}$ from 1.5 T scanner in hospital B. Furthermore, RF magnetic fields were 0.09 microTesla $(\mu \mathrm{T})$ and $0.2 \mu \mathrm{T}$ respectively. In this study, quality performance of the scanners was evaluated based on image quality and apparent diffusion coefficient (ADC). Furthermore, a comparison of hazards and risks was drawn between the two facilities, one with a 1.5 and the other with a 3.0 T MRI scanner, to assist in developing a health and safety programme for the MRI units.

\section{Methodology}

The study was observational-descriptive in nature. An observation tool was used to collect risks and hazardrelated information in the MRI units. To ensure validity, a checklist consisting of all possible risks and hazards was designed according to the ACR manual on MR safety [4]. A walk-through survey was conducted to observe possible existing hazards as well as risks in zones III and IV of the MRI units. A discussion was also conducted with the medical physicists and maintenance engineers as part of the walk-through survey of the maintenance interval of the MRI scanners and the presence of safety guidelines in the MRI units. Since the safety zones were not demarcated in both units, and there was no 5-gauss line in zone IV of hospital B, the MR safety manual [4] was used with the assistance of medical physicists to crosscheck were safety zones and gauss line supposed to be demarcated. Each unit is assigned with two MR radiographers stationed in the control console (zone III) most of the time. However, there are other professionals, e.g. doctors or nurses who bring patients for scanning, porters who transport patients to and from the scanner room, and cleaning personnel responsible for cleaning the units. The information recorded on the checklist was fed into a health and safety baseline risk assessment. Results of quality control tests performed by medical physicists in both hospitals also were reviewed.

\subsection{Data collection tool}

\subsubsection{Checklist}

An observational checklist was designed as part of a tool used to identify the presence of hazards in zone III (control room) and IV (scanner room) of hospital A (Fig. 1) and hospital B (Fig. 2). The checklist consisted of three columns: a brief description of hazards, a column for yes, and a column for no. Descriptions of the hazards found in the checklist were compiled based on safety requirements stipulated in the ACR guideline of 2020. The focus was on the occupational safety requirements. The hazardrelated items contained in the checklist included factors such as the presence of ferromagnetic detectors, the use of hearing protection devices by MR and non-MR personnel, demarcation of the 5-Gauss line, and four MRI zones, as well as lockers to store personal belongings of MR staff. Other factors included in the checklist were the screening 
Fig. 1 1.5 T MRI unit layout



Fig. 2 3.0 T MRI unit layout

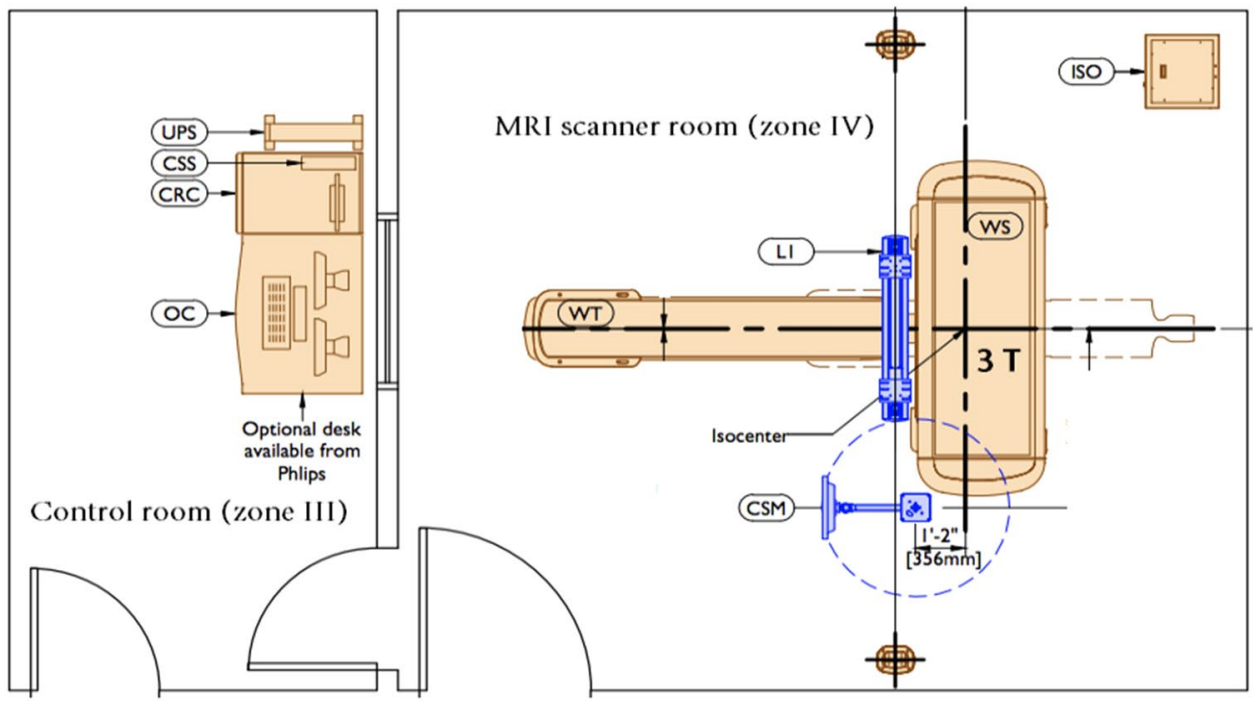

of non-MR personnel entering zones III and IV, health and safety policy, exposure of staff to MRI-related electromagnetic fields, access control, as well as "final stop and final check" requirements.

\subsubsection{Discussion on maintenance intervals and presence of safety guidelines}

A discussion was held with two maintenance engineers of two service providers (Philips and Siemens) to check 
the maintenance interval of the MRI scanners and validate with maintenance schedules. During a walk-through survey, the engineers were asked how often the MRI scanners were serviced. The previous maintenance schedules were provided to check if they corroborated with the answers given during the walk-through survey discussion. Both engineers indicated that they performed quality assurance tests four times annually, followed by a comprehensive service of the scanners every second year. During acceptance testing, a phase mapping technique is used to assess the magnetic fields homogeneity, and the MRI static fields safety assessment is also performed using a calibrated THM1176 3-Axis hall magnetometer. The medical physicists from hospitals $A$ and $B$ use the techniques described below to test the system's performance by assessing image quality and ADC separately on the scanners. The Medical physicists from both hospitals were asked to provide the results of the quality control tests performed on MR scanners in their respective hospitals.

\subsubsection{Presentation of weekly quality control test results}

Quality control procedures were performed on two MR scanners in hospitals A and B. In hospital A, weekly quality control tests were performed on a $3.0 \mathrm{~T}$ Philips Inguena using an ACR phantom that contains structures for measuring geometric accuracy, high-contrast spatial resolution, slice thickness accuracy, slice position accuracy, image intensity uniformity, percent signal ghosting, and low-contrast detectability. In hospital B, tests only were performed for apparent diffusion coefficient. Therefore, quality control tests of two $1.5 \mathrm{~T}$ scanners in hospitals $\mathrm{A}$ and $B$ could not be compared since $1.5 \mathrm{~T}$ in hospital $A$ has been discontinued. Quality control tests on the 3.0 T scanner had been performed once in February 2018 (no quality control data could be provided for subsequent months). The apparent diffusion coefficient test results of the 1.5 T (Siemens, MAGNETOM) for 2018 were compared with the newly established limit values of 2018. The ACR phantom used to perform tests had an inner length of $148 \mathrm{~mm}$ and diameter of $190 \mathrm{~mm}$, filled with a solution containing 10 $\mathrm{mM} \mathrm{NiCl}_{2}$ and $75 \mathrm{mM} \mathrm{NaCl}$.

On a 3.0 T Philips scanner, phantom images were analyzed according to ACR instructions, and the results were compared with the ACR specifications. Geometric accuracy was determined by measuring known distances in the images, while high contrast spatial resolution was evaluated visually based on hole-array pairs with hole diameters of $0.9 \mathrm{~mm}, 1.0 \mathrm{~mm}$ and $1.1 \mathrm{~mm}$. Image intensity uniformity was calculated from pixel values inside the region of interest in a slice containing uniform material, and ghosting values were calculated from the region of interest placed outside the phantom in the image. Furthermore, low-contrast object detectability was visually assessed and this was done by calculating the number of objects visible in images with gradually decreasing contrast and object size. Slice positioning accuracy was measured based on wedge visualization and slide thickness was calculated from a known ramp angle.

Since hospital B performs the diffusion-weighted imaging (DWI), the ADC was conducted on a 1.5 T scanner (Siemens), and the protocol was applied with the scanner operating in a regular mode $\left(25 \mathrm{mT} \mathrm{m}^{-1}\right.$ and slew rate of $40 \mathrm{~T} \mathrm{~m}^{-1} \mathrm{~s}^{-1}$ ). The phantom was secured within the head coil and left in the magnet bore for few minutes, before the scanning procedure. Diffusion sensitization was applied independently in the three orthogonal directions; superior-inferior (SI), anterior-posterior (AP), and right-left (RL), and the directional ADC image was calculated. The ADC measurements were obtained at the center of the images, with a region of interest (ROI) of $20 \times 20$ pixels. The spherical phantom $(10 \mathrm{mmol}$ nickel chloride solution containing $45 \mathrm{mmol}$ sodium chloride to simulate biological conductivity) was kept inside the scanner room at all times to minimize the movement and to acclimatize it to the temperature of the environment. The ADC measurements were recorded for a given $\mathrm{ROI}$ on the $A D C$ map. Furthermore, ADC values were calculated automatically by the software and displayed as a parametric map that reflected the degree of diffusion of water molecules. Images were acquired at $b=1000 \mathrm{~s} / \mathrm{mm}^{2}$.

\subsubsection{Development of base-line risk assessment tool}

Risk assessment was designed using data obtained from an observational checklist to review existing control measures. The risk assessment template consisted of sections that required descriptions of tasks, hazards, risks, an indication of whether a hazard is related to health or safety, consequence, probability, risk rating, and outcome, existing control measures, as well as emergency action required. The probability and consequence scales were adopted from the Exeter MRI risk classification scheme [9]. The probability scale ranged from "improbable" on a scale of one to "very likely" classified as five, while consequence ranged from a classification scale of one as "minimal effect" to five as "irreversible effect". To determine risk rating, the probability score was multiplied by the consequence score and this resulted in the determination of the overall risk outcome. The risk outcome was classified in terms of high, moderate, low, or no risk. The derivation of the overall risk score was based on the outcome or total score of the risk rating. Regarding the score values for risk outcome, if the risk rating was $>15$, the risk outcome was classified as high, $>5 \leq 15$ was moderate, $\leq$ five was low risk, and zero being no risk. The emergency action required to improve 
controls was determined by the risk rating score; a score factor of $>$ five led to the recommendation of emergency action to improve safety controls.

\section{Results}

\subsection{Risk assessment}

This study reports on the results of a baseline risk assessment conducted in the MRI units of two hospitals housing two different strengths of MRI scanners. Hospital A's 3.0 T MRI unit had a total of ten safety risks and four health risks, whereas nine safety risks and three health risks were identified from the 1.5 T MRI unit in hospital B. In both hospitals, factors such as the demarcation of four safety zones, presence of ferromagnetic detectors, and performance of final stops and checks of personnel entering the scanner room (zone IV) were identified as high risks (>15), since they either did not exist, were very likely to pose health and safety risks to MR and non-MR staff, or were not in accordance with the requirements of the ACR guideline of 2020 (Tables 1 and 2). Moderate risks (> $5 \leq 15$ ) also were identified in both hospitals, and these were classified based on the observational probability that they could occur and existing control measures in place to mitigate the risks. Low risks $(\leq 5)$ were classified only if the probability of risk was observed to be minimal. Demarcation of the 5-gauss line, access control, lockers for MR and non-MR staff to store their belongings, workers' exposure to MRIrelated electromagnetic fields, visibility of a health and safety policy, use of hearing protectors during patients' examination, safety screening, and a red illuminated sign, reading Magnet is always $\mathrm{ON}$, were rated moderate risks in both hospitals.

Hospital A had a low-risk classification on fading of the 5-gauss line demarcation on the floor of zone IV, and this influenced the health and safety overall risk rating of the hospital (A). The health and safety in the MRI units of both hospitals were rated moderate risks ( $>5 \leq 15)$, and emergency control measures required to relegate the risk score to low risk $(\leq 5)$ are indicated in Tables 1 and 2 .

Hospital B scored an average risk rating of 13.1 and was classified as moderate risk. A factor that influenced the risk-rating score included the lack of demarcation of the fourth MR safety zone. Through a walk-through survey, it was observed that none of the four MR safety zones was labelled accordingly and this could make it difficult to identify zones, especially to non-MR staff and newly appointed MR staff who had not received in-house MR safety training. The ferromagnetic detector was not installed, there was no final stop and checks performed on non-MR staff entering zone III and IV, and a 5-gauss line was not marked on the floor in zone IV. This does not comply with the latest requirements of the ACR MR safety rules. Although some factors were classified as a moderate risk, appropriate and substantial alternative control measures were used to reduce the identified health and safety risks

Hospital A had a low-risk score on the 5-gauss line, influencing the average risk rating of the entire MRI unit to 12.3 (moderate risk). Similar to hospital B, hospital A had noncompliant factors that influenced the risk-rating scores to high risks. Such factors included a non-functioning ferromagnetic detector, non-demarcated MR safety zones, and final stop and checks. The majority of risks observed were safety-related, which is a major concern in terms of ACR MR Safety Guidelines. However, it was noted that the majority of control measures in place were acceptable and capable of reducing the identified health and safety risks to low and moderate

\subsection{Quality control}

\subsubsection{A 3.0 T Philips Inguena}

On a 3.0 T scanner, the results of quality control for geometric accuracy, high spatial resolution, slide thickness, and slice position accuracy are illustrated in Table 3. Image intensity uniformity, percentage signal ghosting, and low contrast object detectability were also determined, and a summary of the results are found in Table 4.

The geometric accuracy test was performed to assess whether geometric distortion could occur during the scanning process. The phantom dimensions in the image were similar to the true dimensions. As prescribed by the ACR procedure, the phantom dimensions were measured in the localizer, slice 1 and slice 5 of the axial series and the localizer presented an accuracy of $146.2 \mathrm{~mm}$. The absolute value for the percentage of geometric distortion did not exceed $2 \%$ (Fig. 3b). The real inner diameter of the phantom was $190 \mathrm{~mm}$ and the inside end-to-end length was $148 \mathrm{~mm}$, meeting standards of the ACR quality control guidance, which allows for $\mathrm{a} \pm 2 \mathrm{~mm}$ deviation. With a resolution insert located in slice 1 , a high contrast spatial resolution test was conducted to determine the scanner's ability to resolve small high-contrast objects within proximity of each other. Furthermore, the field of view and matrix size for the axial series were chosen to produce a resolution of $1.0 \mathrm{~mm}$ in both directions (Fig. 3a). The measured resolution of both axial series was $1.0 \mathrm{~mm}$ in both directions.

On both ACR series, the measured slice thickness was $5.6 \mathrm{~mm}$ and $5.3 \mathrm{~mm}$, resulting in the scanner passing the slice thickness test $(5.0 \mathrm{~mm} \pm 0.7 \mathrm{~mm}$ ) (Fig. 2a). The radiofrequency (RF) amplifier nonlinearity did not cause any distorted RF pulse shapes and no thickness error was 


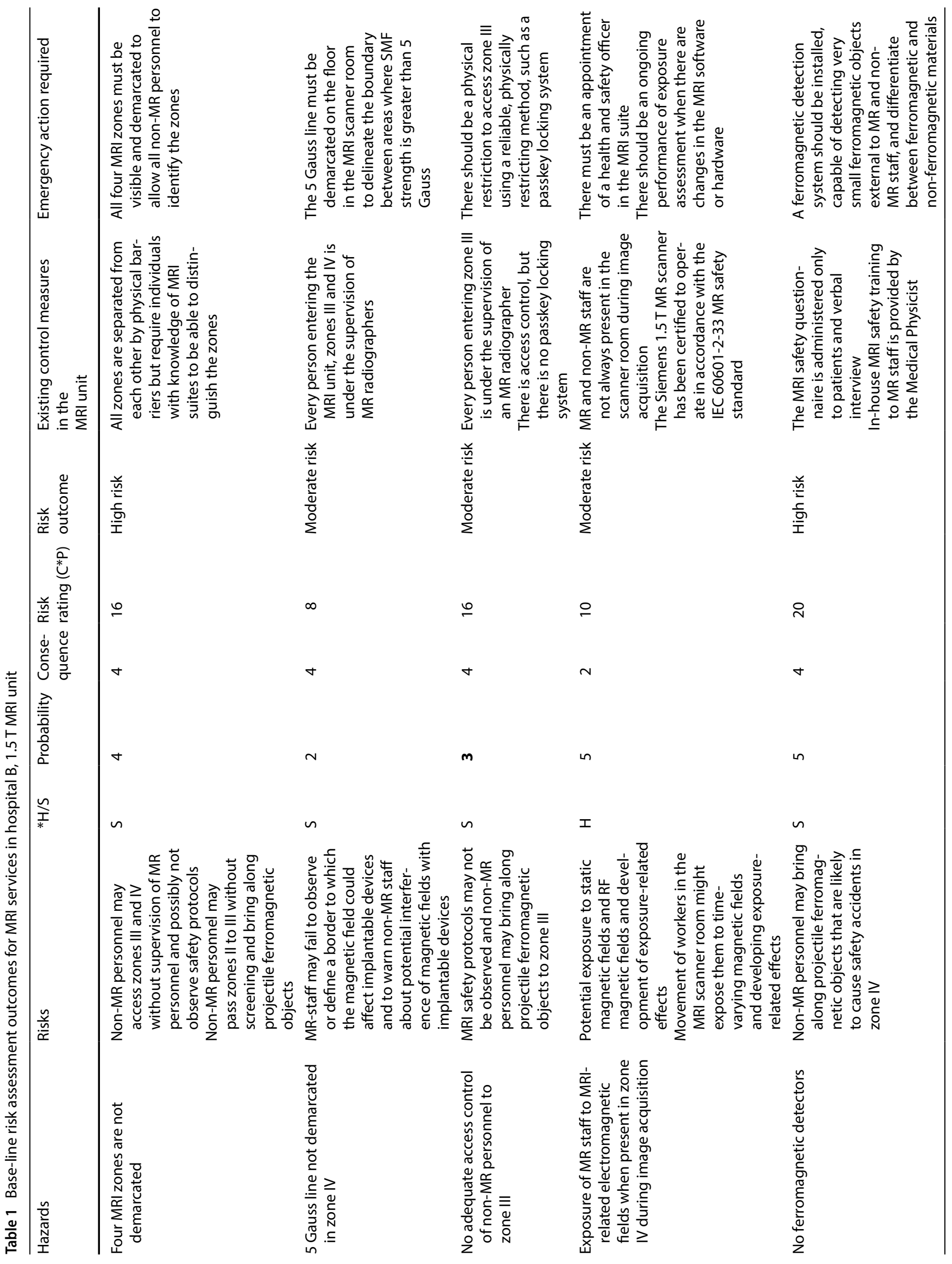




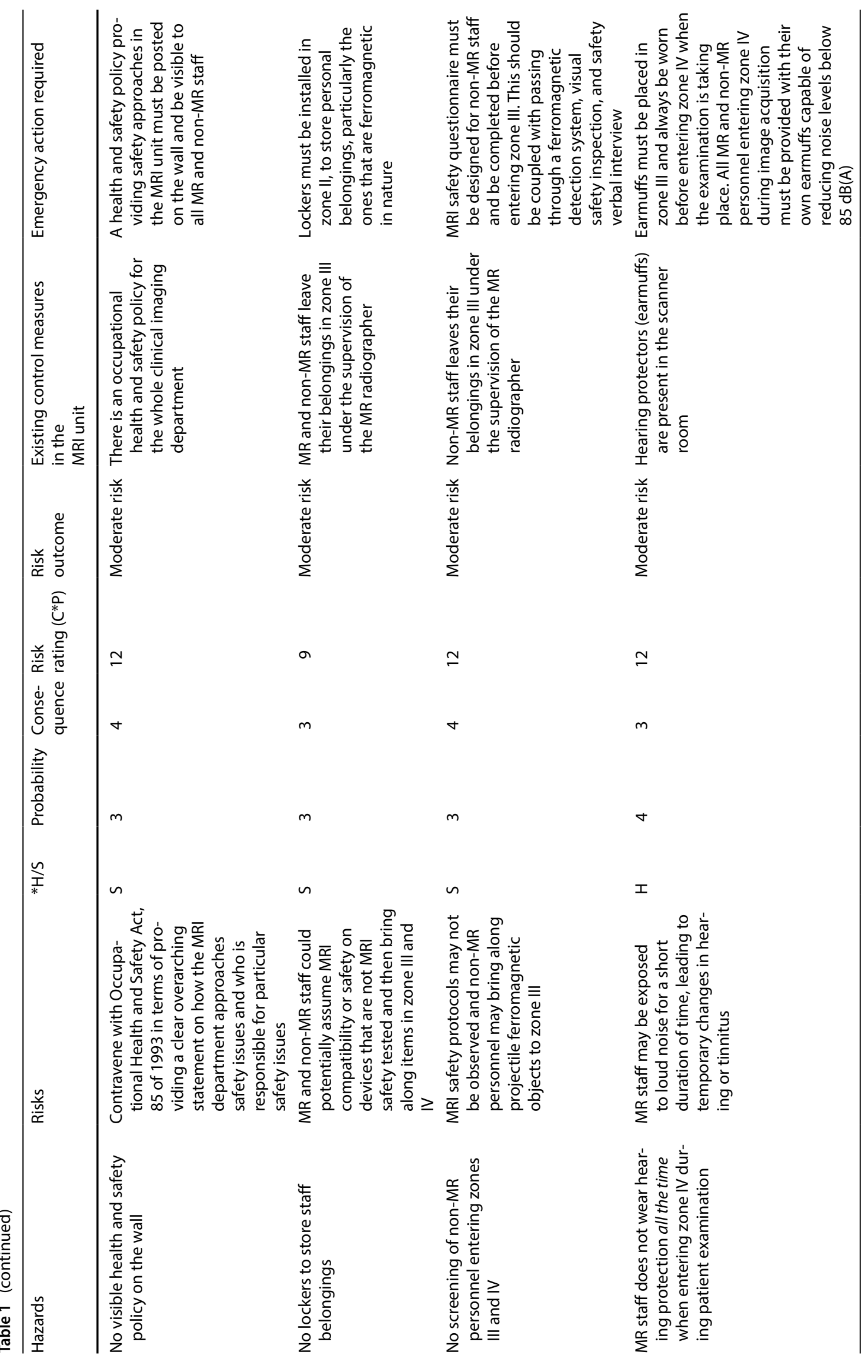




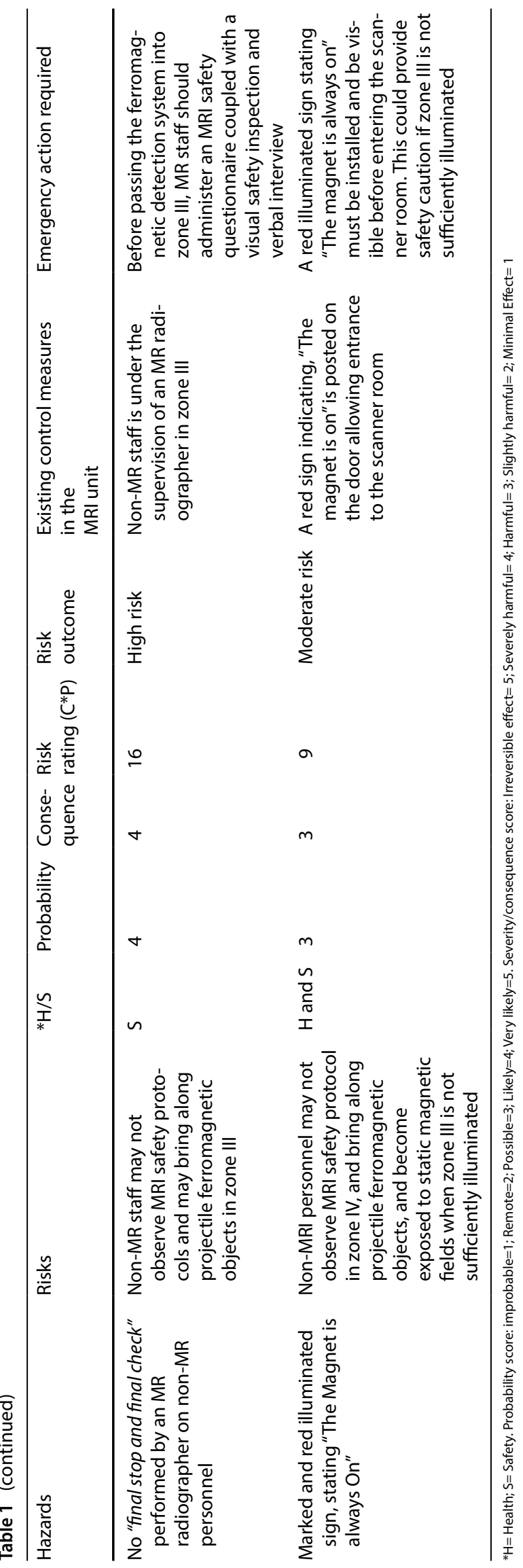

SN Applied Sciences

a SPRINGer Nature journal 







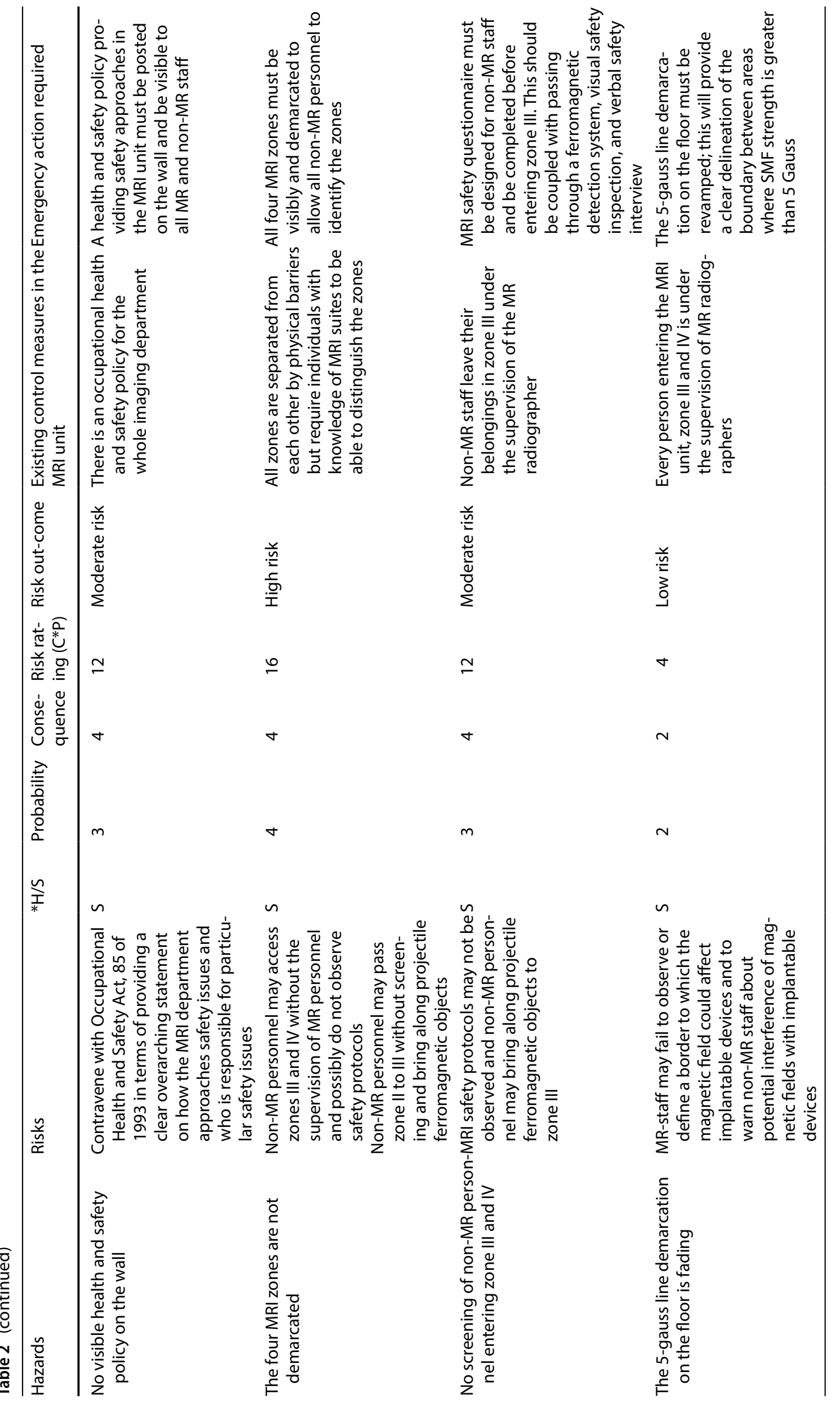

SN Applied Sciences 


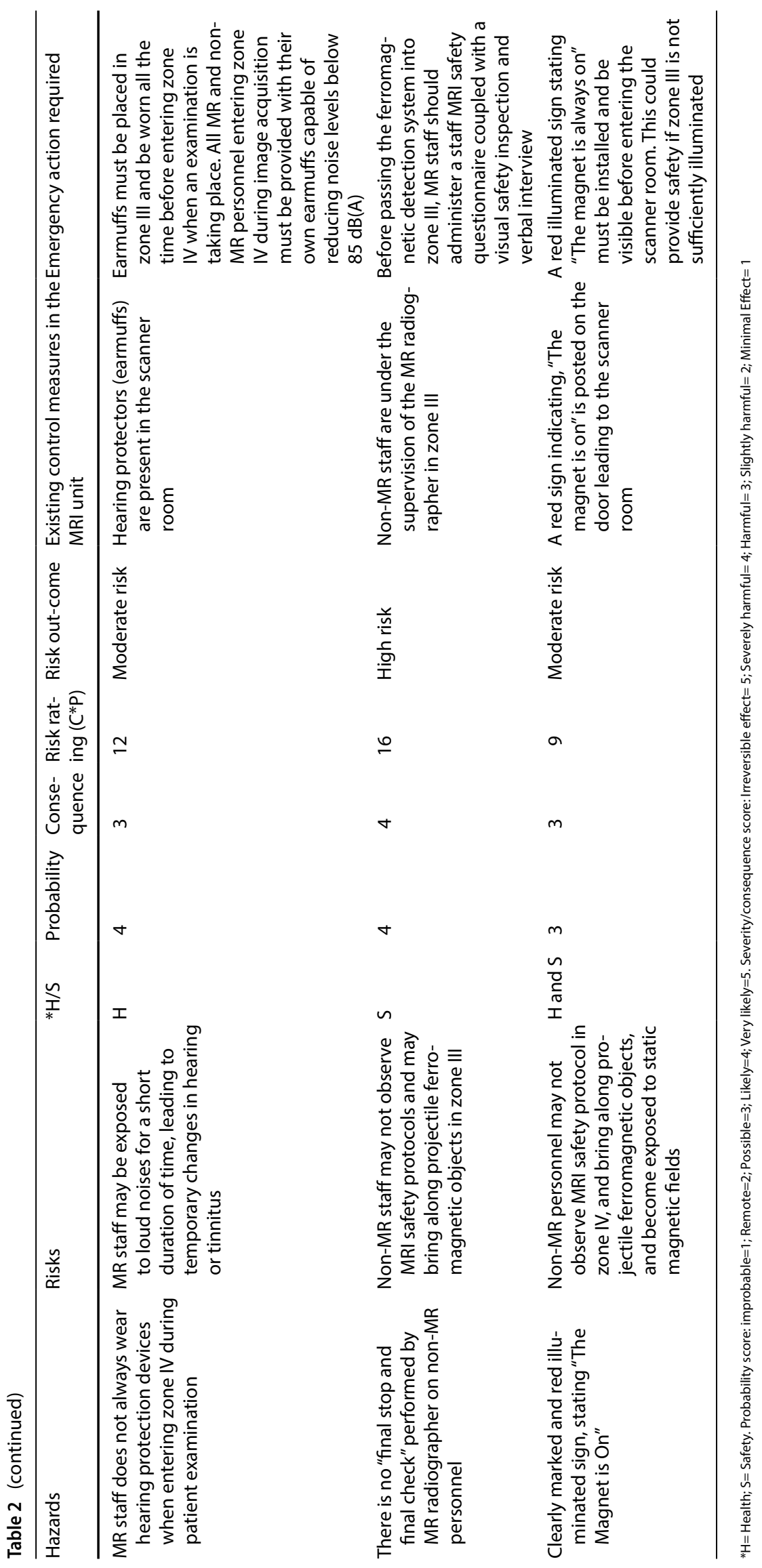


identified on either ACR series. The absolute bar length difference for slice 1 and slice 11 (Fig. 4b) for position accuracy was less than $5 \mathrm{~mm}$.

The high and low signal levels within a large water-only region of the phantom were measured for both ACR T1 and $\mathrm{T} 2$ series. To obtain the region of lowest signal in the $\mathrm{ROI}$, the display window was set to its minimum and the level was lowered until the entire area inside the large ROI was white. The levels were raised until a small region ( 1 $\mathrm{cm}^{2}$ ) of white pixels remained inside a large ROI to obtain the highest signal. The percentage Integral uniformity (PIU) for ACR T1 was 85.3 and $78.2 \%$ for ACR T2, failing the test (Table 4).

To determine the ghost ratio, five intensity measurements were made for the ACR 11 series; the average intensity in the image of the phantom, as well as the average intensity in the background at four locations outside of the phantom. The ghosting ratio was 0.00678 , being less than the acceptable ghost ratio value of less than or equal to $0.025(2.5 \%)$.

Measurements were made for ACR T1 and T2 series using four slices for low contrast detectability. In each slice, the low contrast objects appeared as rows of small disks, from the center of a circle. All the disks on each slice had the same level of contrast. In order, the contrast values were $1.4 \%, 2.5 \%, 3.6 \%$, and $5.1 \%$ (Fig. $5 \mathrm{~b}$ ). All the disks in a given spoke had the same diameter. The measurements comprised counting the number of complete spokes seen in Fig. 5b. The ACR T1 had a total score of 40 spokes, while T2 failed with a total of 29 spokes (ideal $\geq 37$ ).

\subsection{A 1.5 T Siemens MAGNETOM}

The ADC measurements were taken when the phantom acclimatized to an approximate temperature of $21^{\circ} \mathrm{C}$; however, the calculated average temperature for the period of 2018 when measurements were performed was $22.8^{\circ} \mathrm{C}$. The measured average ADC values in the same period was $0.00204 \mu \mathrm{m}^{2} / \mathrm{s}(\mathrm{Std} \pm 0.000132)$, this was on the computed values for $\mathrm{X}, \mathrm{Y}$ and $\mathrm{Z}$. In order to establish the $A D C$ values, each non-zero $b$-value (on the ADC maps) was computed using formula (1):

$\mathrm{ADC}_{\mathrm{b}}=\frac{1}{b} \ln \left[\frac{S_{0}}{S_{b}}\right]$

where $S_{0}$ is the $b=0$ image and $S_{b}$ is the isotropically weighted DWI at the given b-value. The calculated ADC values for $S_{0}, S_{b}, X, Y$, and $Z$ are found in Table 5 .

The ADC values were well within the acceptable range; however, it was noted that some measurements were influenced by varying temperatures on certain days (Fig. 6). None of the measurements were below the low acceptable limit.

On day 57 of the measurements, the ADC values for $X$, $Y$, and $Z$ were $0.00244 \mu \mathrm{m}^{2} / \mathrm{s}, 0.00243 \mu \mathrm{m}^{2} / \mathrm{s}$, and 0.00246 $\mu \mathrm{m}^{2} / \mathrm{s}$ respectively. These values were noted to be higher than the acceptable upper limit and the recorded room temperature was $37.7^{\circ} \mathrm{C}$. The temperature rise was influenced by the defective air-cooling system in the scanner room. After maintenance of the air-cooling system, the measured $A D C$ values for $X, Y$ and $Z$ were significantly lower: $0.00188 \mu \mathrm{m}^{2} / \mathrm{s} 0.00186 \mu \mathrm{m}^{2} / \mathrm{s}$ and $0.00189 \mu \mathrm{m}^{2} / \mathrm{s}$,

Table 3 Summary of results for geometric accuracy, high spatial resolution, slide thickness, and slice position accuracy on a $3.0 \mathrm{~T}$ Philips scanner

\begin{tabular}{|c|c|c|c|c|c|c|c|c|c|c|c|c|c|}
\hline & & \multicolumn{7}{|c|}{ Geometric accuracy } & \multicolumn{5}{|c|}{ High spatial resolution } \\
\hline & & \multicolumn{2}{|c|}{ Vertical (mm) } & \multicolumn{2}{|c|}{ Horizontal (mm) } & $45^{\circ} 325^{\circ}$ & Limit & Test status & \multicolumn{2}{|c|}{ Vertical (mm) } & $\begin{array}{l}\text { Horizon- } \\
\text { tal (mm) }\end{array}$ & Limit & Test status \\
\hline \multicolumn{2}{|c|}{ ACR T1 Slice 1} & 189 & .32 & 189.92 & & & $190 \pm 2 \mathrm{~mm}$ & Pass & 1 & & 1 & $\leq 1$ & Pass \\
\hline \multicolumn{2}{|c|}{ ACR T1 Slice 5} & $18 \varepsilon$ & 3.73 & 189.02 & & $\begin{array}{l}{ }^{*} 188.32 \\
189.24\end{array}$ & $190 \pm 2 \mathrm{~mm}$ & Pass & 1 & & 1 & $\leq 1$ & Pass \\
\hline \multirow[t]{3}{*}{ Localize } & & 146 & & & & & $148 \pm 2 \mathrm{~mm}$ & Pass & & & & & \\
\hline & \multicolumn{7}{|c|}{ Slide thickness } & \multicolumn{6}{|c|}{ Slice position accuracy } \\
\hline & Top $(n$ & & Bottom (mm & n) $\mathrm{ST}$ & Limit & Test st & & Left (mm) & Right (mm) & Differ & rence $(\mathrm{mm})$ & Limit & Test status \\
\hline \multirow[t]{2}{*}{ ACR T1 } & 79.92 & & 44.45 & 5.6 & $5 \pm 0.7 \mathrm{~mm}$ & n Pass & Slice 1 & 33.35 & 32.22 & -1.13 & & $\leq 5$ & Pass \\
\hline & & & & & & & Slice 11 & 31.32 & 28.62 & -2.7 & & $\leq 5$ & Pass \\
\hline \multirow[t]{2}{*}{ ACR T2 } & 98.63 & & 36.12 & 5.3 & $5 \pm 0.7 \mathrm{~mm}$ & n Pass & Slice 1 & 30.66 & 33.6 & 2.98 & & $\leq 5$ & Pass \\
\hline & & & & & & & Slice 11 & & & 0 & & $\leq 5$ & Pass \\
\hline
\end{tabular}





(a) High spatial resolution


(b) Geometric accuracy

Fig. 3 a High spatial resolution, b Geometric accuracy

with a measured room temperature of $15.9^{\circ} \mathrm{C}$. Also, on day 94 , the measured room temperature was $30.6^{\circ} \mathrm{C}$ and the $A D C$ values for $X\left(0.00232 \mu \mathrm{m}^{2} / \mathrm{s}\right), Y\left(0.00232 \mu \mathrm{m}^{2} / \mathrm{s}\right)$, and $Z\left(0.00236 \mu \mathrm{m}^{2} / \mathrm{s}\right)$ were significantly higher, above the acceptable upper limit.

\section{Discussion}

\subsection{Base-line risk assessment}

There is a dearth of literature on the assessment of health and safety risks in the clinical MRI units, and this is the first study in South Africa that evaluated risks related to occupational health and safety in the MRI units of two public hospitals. The results show that some similar risks and hazards exist in the MRI units of hospitals A and B. A relatively small number of high risks were classified compared to moderate and low risks in both hospitals. However, it was noted that the overall risk rating (safety performance) was relatively lower in hospital A (12.3) compared to hospital $B$ (13.1). This has been influenced by the demarcation of the 5-gauss line on the floor of zone IV (scanner room). Hospital A scored a risk rating of four (low risk) due to a fading 5-gauss line on the floor, while hospital B had no demarcated line to provide delineation of the boundary between areas where static magnetic field (SMF) strength is greater than 5-gauss. The American experts on MR safety indicated that in areas where SMFs' strength exceeds 5-gauss, they should be marked as potentially hazardous $[6,10,11]$. Restoring the 5 -gauss line in hospital A, therefore is necessary to relegate the overall risk rating score.

It was noted from the outcome of the risk assessment that hospital B did not have a ferromagnetic detector and this contravenes with the safety requirements of $A C R$. The ACR MR Safety Committee (2020) requires all MRI facilities to have a ferromagnetic detector before one may enter zone III, and the use of such a detector should be coupled with a visual safety inspection. Apart from screening using ferromagnetic detectors, Keevil [10] recommends that MR facilities must establish and mark the four MRI zones, have strict access control, and ensure safety screening before entering zone III [12]. These factors were observed to constitute moderate risks in both hospitals. Improper use of hearing protectors and absence of red illuminated signs, indicating, "Magnet is always on" was noted to be a risk. Heismann et al. [13] maintain that the switching of magnetic field gradients creates a vibration that generates acoustic noise and patients would experience an average sound pressure level ranging from 80 to $109 \mathrm{~dB}$. 



(a) Slide thickness



(b) Slice positioning accuracy

Fig. 4 a Slide thickness; and b Slice positioning accuracy

The safety risks including physiological effects encountered in the MRI units are summarized in Table 6 and were adopted from the work of Schaap [14]. To reduce exposure to acoustic noise and avoid hearing impairments, Kraff and Ladd [3] recommend the use of earmuffs or earplugs during patients' scanning. Regarding illuminated signs, Sammet [2] purports that special warning signs indicating the strong magnetic field and its associated hazards must be present in MRI facilities. In both hospitals MR safety signs are found indicating the magnet is always on, however, they are not illuminated. This might be a safety risk especially when zone III is not properly illuminated.

Apart from ACR non-compliance safety parameters, several studies describing the potential effects of MRI-related
EMFs on patients' and healthcare workers' health and safety are provided in Table 6 . The MRI-related EMFs are severely hazardous in zone IV, particularly near the scanner. Similar risks presented in Table 6 also exist in the MRI units included in this study; however, their assessment was beyond the scope of this study. Both facilities do not have a visible MR safety policy in place. However, it was noted during a walk-through survey that there was a health and safety policy for the entire clinical imaging department. Similar MR safety non-compliance has also been noted in a Ghanaian study [24]. Cross et al. [5] and Kanal et al. [6] suggest that MRI facilities must have specific and updated MR safety policies and this was not found during the observations made in both hospitals. Since both facilities have no 
Table 4 Summary of results for image intensity uniformity, percentage signal ghosting, and low contrast object detectability on a $3.0 \mathrm{~T}$ Philips scanner

Image intensity uniformity

\begin{tabular}{|c|c|c|c|c|c|}
\hline & High signal ROI & Low signal ROI & PIU (\%) & Limit 3T (\%) & Test status \\
\hline ACR T1 & 1672.58 & 1244.38 & 85.3 & $\geq 82$ & Pass \\
\hline ACR T2 & 1644.56 & 1055.83 & 78.2 & $\geq 82$ & Fail \\
\hline \multicolumn{2}{|c|}{ Percentage signal ghosting } & \multicolumn{4}{|c|}{ Low contrast object detectability } \\
\hline Parameters & Values & Slice & Spokes T1 & Spokes T2 & Ideal \\
\hline Large ROI & 1494.44 & 8 & 10 & 9 & \\
\hline Top ROI & 2.09 & 9 & 10 & 10 & \\
\hline Bottom ROI & 22.89 & 10 & 10 & 10 & \\
\hline Left ROI & 2.71 & 11 & 10 & & \\
\hline Right ROI & 2.01 & & & & \\
\hline GR & 0.00678 & & & & \\
\hline Limit & $\leq 0.025$ & Total & 40 & 29 & $\geq 37$ \\
\hline Status & Pass & & Pass & Fail & \\
\hline
\end{tabular}

strict access control to zones III and IV, there is a likelihood of exposure to MRI-related EMFs among non-MR and MR staff who enter the scanner room, and increased odds of experiencing transient health effects. To reduce these risks, both facilities need to adhere to the ACR guidance document on MR safe practices [25].

\subsection{MR quality control}

A comprehensive assessment of the performance of two MRI scanners was provided in this study. A 3.0 T scanner complied with the majority of the image quality features that were assessed. However, image intensity uniformity and low contrast objectivity did not meet the ACR acceptable criteria. The 1.5 T scanner performed well with regard to $A D C$ and the majority of measurements were within the low and upper acceptable limit. A defective air-cooling system in the 1.5 T scanner room resulted in elevated temperatures that influenced the ADC measurements. The current study showed that under normal, controlled room temperature, the ADC measurements were stable and within the acceptable range, and this accords with the results obtained from a study that compared the DWI results using a phantom [26]. However, since the phantom was stored in the scanner room before ADC measurements, the room temperature was unstable, and significantly high ADC measurements were obtained. Lavdas et al. [27] have shown that the stability of ADC measurements could be attained when the phantom is stored in temperature-controlled environments.
The ACR T2 failed the low contrast object detectability with 29 spokes while the PIU for image intensity uniformity was $78.2 \%$, lower than the acceptable ACR quality control (QC) criterion. According to Chen et al. [28], failure in a uniformity test could potentially be caused by poor phantom positioning, head coil failure, and ghosting. The vibration of an unstable phantom could create ghosting signals that influence the image intensity. In this study, failure in the low contrast detectability test could have been caused by intermittent ghosting or a tilting phantom. The persistence of this failure would require a medical physicist to repeat the tests and if it continues, a maintenance engineer should be consulted to establish the cause. Since poor signal uniformity suggest that the RF coil has a significantly greater variation in image intensity than normal [29], failure of the image uniformity test in this study could have been caused either by a defective RF coil or by underlying RF subsystem faults.

Our previous study [8] recommended the development of a health and safety programme to manage peak exposure levels, especially in the areas of zone IV where MRI-related activities are performed. The development of such a comprehensive programme required the existing control measures in the zone III and IV to be reviewed, and the scanners' performance based on weekly quality control protocols be evaluated. Based on the results of this study, control measures employed to mitigate the peak exposure levels described previously [8] seemed not to exist. 


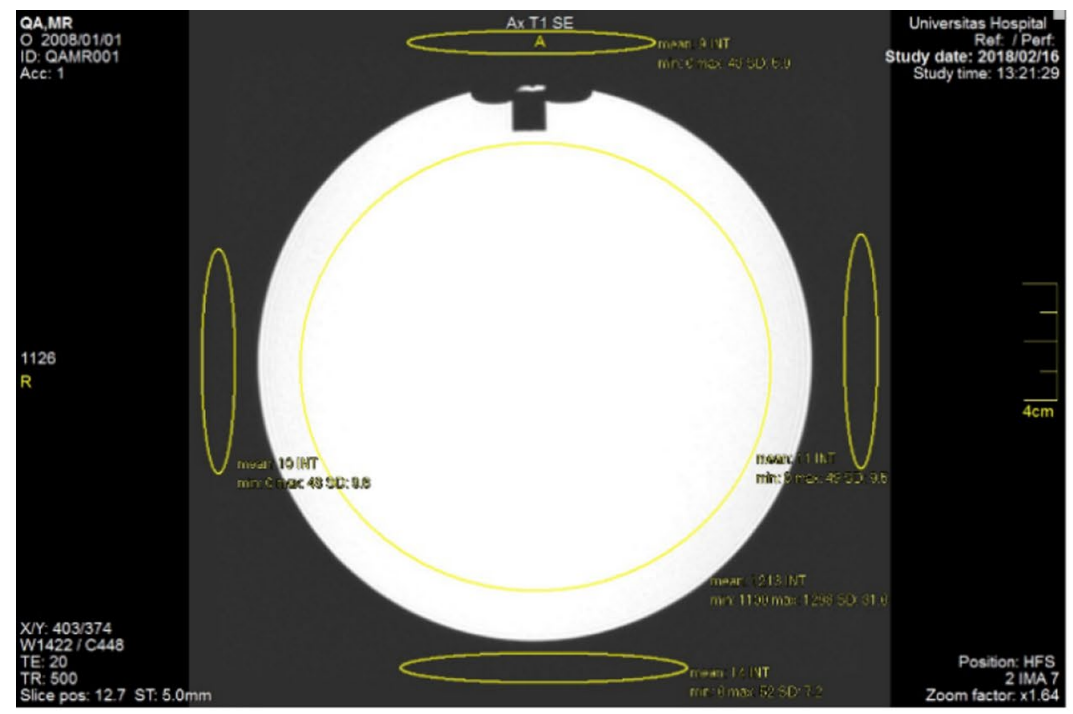

\section{(a) Percentage signal ghosting}
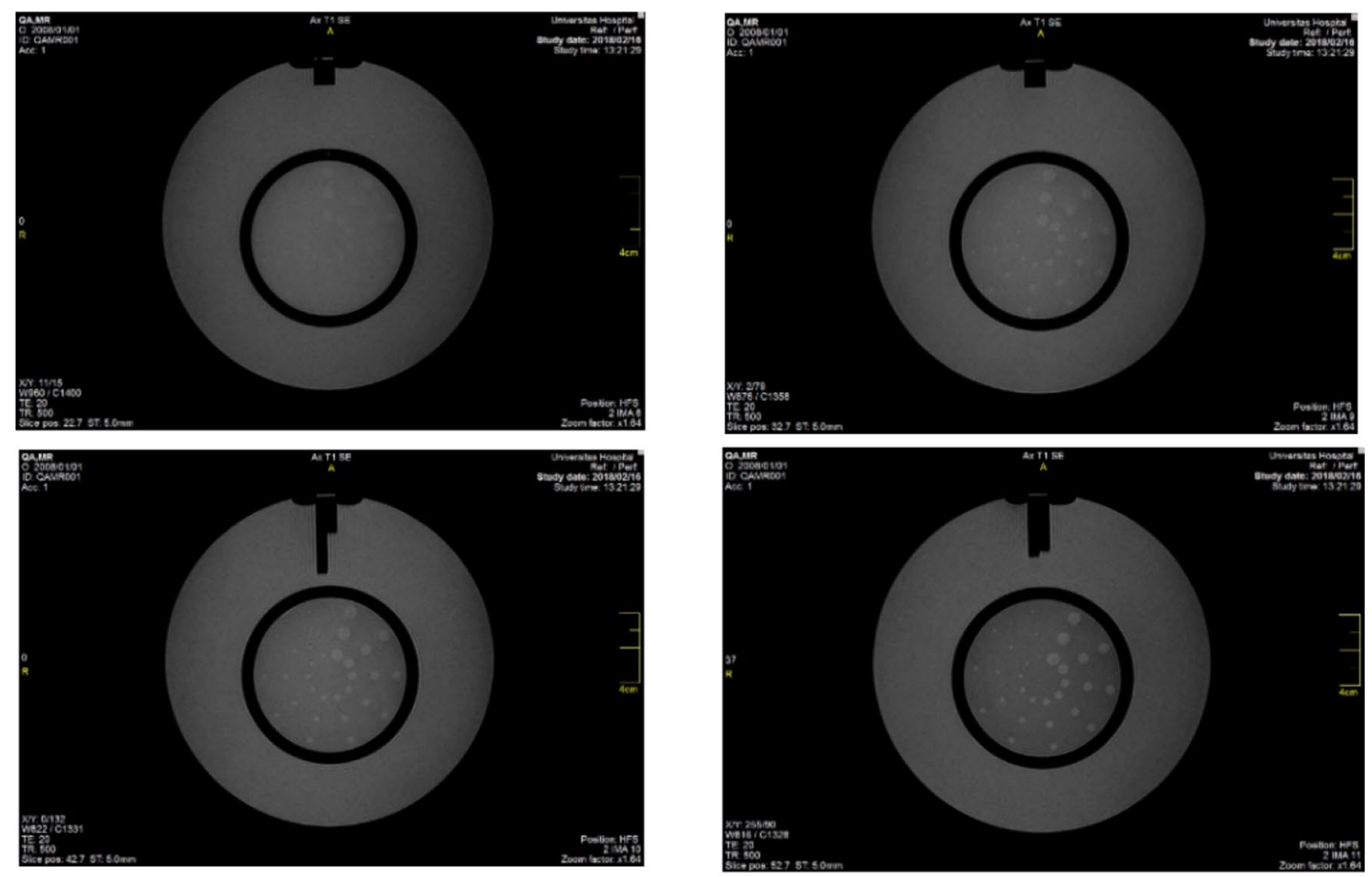

\section{(b) Low object detectability}

Fig. 5 a Percentage signal ghosting, Image uniformity intensity, and b Low contrast object detectability

Table 5 The measured ADC values for 1.5 T Siemens

\begin{tabular}{llllll}
\hline$S_{0}$ & $S_{b}$ & $\ln$ & $b$ & \multicolumn{2}{l}{$\begin{array}{l}\text { ADC values } \\
\left(\mu \mathrm{m}^{2} / \mathrm{s}\right)\end{array}$} \\
\hline 1216 & 164.5 & 2.000411492 & 1000 & $\mathrm{Y}(\mathrm{A} / \mathrm{P})$ & 0.00200 \\
1237.2 & 166.3 & 2.006812655 & 1000 & $\mathrm{Z}(\mathrm{S} / \mathrm{l})$ & 0.00201 \\
1244.9 & 165.9 & 2.015425287 & 1000 & $\mathrm{X}(\mathrm{R} / \mathrm{L})$ & 0.00202 \\
\hline
\end{tabular}

SN Applied Sciences

A SPRINGER NATURE journal

\section{Conclusions}

This study showed that many areas within MRI units of both hospitals comply with occupational health and safety requirements. Although the results of risk assessment suggest that moderate risks exist within the facilities, according to the risk classifications, areas of high risk require 


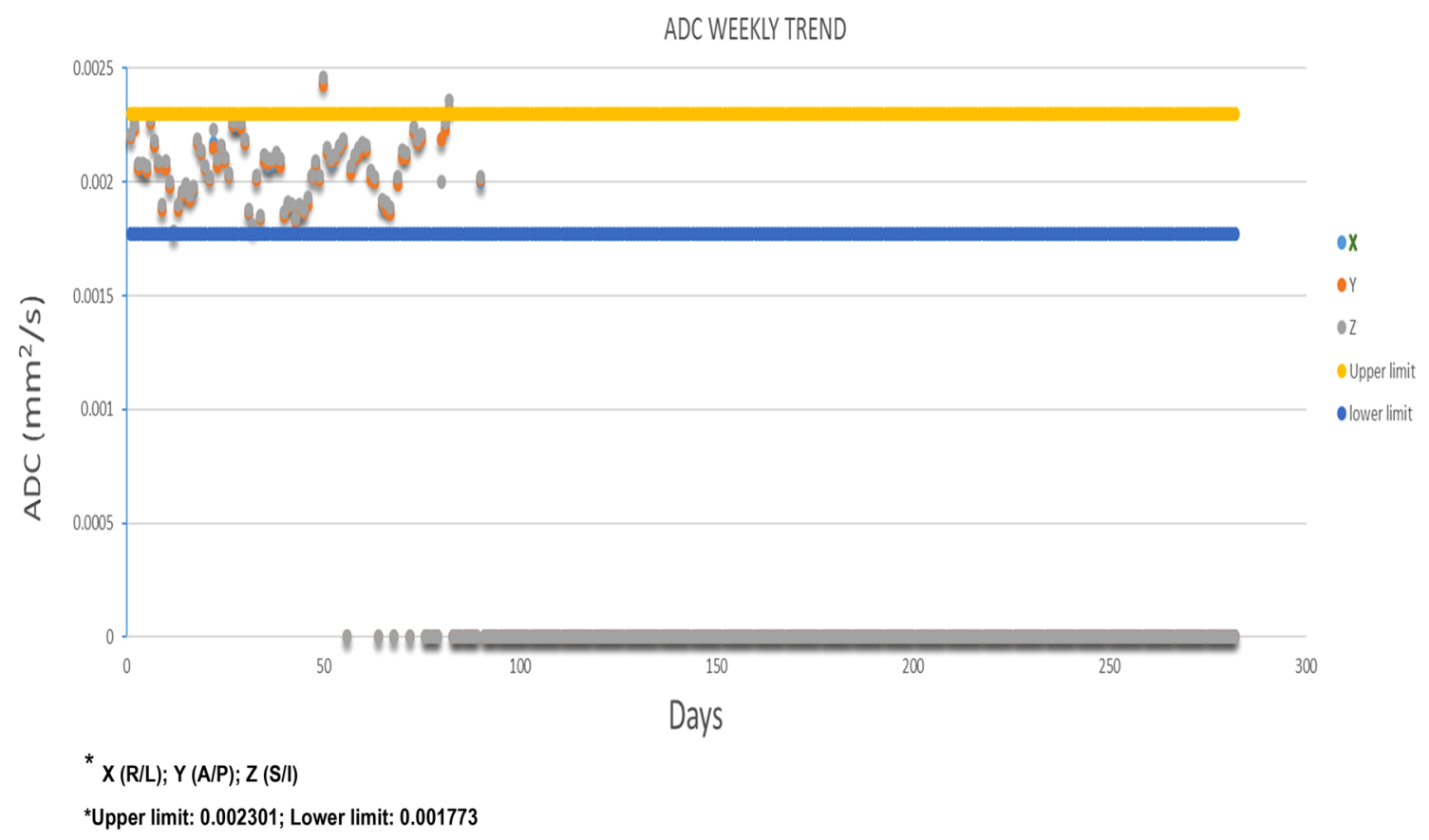

Fig. 6 Apparent diffusion coefficient weekly trends for 2018

Table 6 Comparison of previous studies on MRI health and safety risks

\begin{tabular}{|c|c|c|c|}
\hline Fields & Physiological and acute health effects & Safety risks & Reference \\
\hline SMFs & $\begin{array}{l}\text { Transient symptoms that last for a short } \\
\text { duration of time following exposure }\end{array}$ & $\begin{array}{l}\text { Projectile effects of ferromagnetic } \\
\text { objects }\end{array}$ & $\begin{array}{l}\text { Patel et al. [15], Atkinson et al. [16], } \\
\text { Chaljub et al. [17] }\end{array}$ \\
\hline RF energy & $\begin{array}{l}\text { Thermal-related effects such as tissue } \\
\text { heating }\end{array}$ & $\begin{array}{l}\text { Heating of implantable medical devices } \\
\text { such as cardioverter defibrillator }\end{array}$ & $\begin{array}{l}\text { Nakamura et al. [18], Shellock [19], } \\
\text { Shellock and Crues [20] }\end{array}$ \\
\hline $\begin{array}{l}\text { Time varying } \\
\text { magnetic } \\
\text { fields }\end{array}$ & $\begin{array}{l}\text { Transient symptoms related to cogni- } \\
\text { tive functions in the visual, visuomo- } \\
\text { tor and working memory domains }\end{array}$ & $\begin{array}{l}\text { Disrupting the signal of implanted } \\
\text { pacemakers and neuro-stimulators }\end{array}$ & $\begin{array}{l}\text { Chakeres et al. [21], Capstick et al. [22], } \\
\text { Theysohn et al. [23] }\end{array}$ \\
\hline
\end{tabular}

urgent attention. Such an area is found where the lack of clear demarcation holds a high risk of potential safety accidents, and safety inspections using visual inspections, a safety questionnaire, and ferromagnetic detectors should be employed. Furthermore, potential risks of exposure to MRI-related EMFs in zone IV of the MRI facilities included in this study exist [8]. It is proposed that a comprehensive MRI-specific health and safety programme be developed in line with the results of the current study and the recommendations of our previously published study [8]. The medical physicist who assumes the role of MR safety officer could spearhead such a programme.

To ensure optimal image quality performance of hospital $A$, a maintenance engineer of a respectable MR vendor should be consulted to inspect and service the RF subsystems of the scanner. Furthermore, the stability of the phantom should be maintained at all times to avoid MRI QC test failures. Failure of the air-cooling system in the scanner room of hospital B (1.5 T Siemens) resulted in temperature variability which subsequently influenced the $A D C$ measurements. It has been shown that desired ADC measurements could be best obtained if the measured temperature of the scanner bore is approximately $21^{\circ} \mathrm{C}$ [30]. It is therefore recommended that before performing ADC measurements, it must be ensured that the air-cooling system is working efficiently and the scanner bore temperature does not exceed $21^{\circ} \mathrm{C}$.

Availability of data and material Not applicable.

Code availability Not applicable.

\section{Declarations}

Conflicts of interest The author declares no conflict of interest.

Ethical clearance Prior commencement of this study a written informed consent was obtained from two maintenance engineers. Ethical clearance was obtained from the University of the Free 
State Health Sciences Ethics Committee (Reference Number: UFSHSD2018/0438/3107).

Open Access This article is licensed under a Creative Commons Attribution 4.0 International License, which permits use, sharing, adaptation, distribution and reproduction in any medium or format, as long as you give appropriate credit to the original author(s) and the source, provide a link to the Creative Commons licence, and indicate if changes were made. The images or other third party material in this article are included in the article's Creative Commons licence, unless indicated otherwise in a credit line to the material. If material is not included in the article's Creative Commons licence and your intended use is not permitted by statutory regulation or exceeds the permitted use, you will need to obtain permission directly from the copyright holder. To view a copy of this licence, visit http://creativecommons. org/licenses/by/4.0/.

\section{References}

1. Tsai LL, Grant AK, Mortele KJ, Kung JW, Smith MP (2015) A practical guide to MR imaging safety: What radiologists need to know. Radiographics 35(6): 1722-1737

2. Sammet $S$ (2016) Magnetic resonance safety. Abdom Radiol 41(3): 444-451

3. Kraff O, Ladd ME (2016) MR safety update 2015: Where do the risks come from? Curr Radiol Rep 4(6): 34

4. Safety ACR (2020) Committee on MR Safety. ACR Manual on MR Safety 20201-202056

5. Cross NM, Hoff MN, Kanal KM (2018) Avoiding MRI-related accidents: A practical approach to implementing MR safety. J Am Coll Radiol 15(12): 1738-1744

6. Kanal E, Barkovich AJ, Bell C, Borgstede JP, Bradley Jr WG, Froelich JW, Gimbel JR, Gosbee JW, Kuhni-Kaminski E, Larson PA (Expert Panel on MR Safety) (2013) ACR guidance document on MR safe practices: 2013. J Mag Reson Imag 37(3): 501-530

7. Durbridge $G$ (2011) Magnetic resonance imaging: Fundamental safety issues. J Orthop Sports Phys Therapy 41(11): 820-828

8. Rathebe P, Weyers C, Raphela F (2021) Exposure levels of radiofrequency magnetic fields and static magnetic fields in 1.5 and 3.0 T MRI units. SN Appl Sci 3: 157. https://doi.org/10.1007/ s42452-021-04178-3

9. Benattayallah A (2015) EMRRC Health \& Safety Profile \& Risk Assessment, Exeter: University of Exeter. Available online Access date: 12 November 2020

10. Keevil S (2016) Safety in magnetic resonance imaging. Med Phys $4(1)$

11. Sammet $C L$, Yang $X$, Wassenaar PA, Bourekas EC, Yuh BA, Shellock F, Sammet S, Knopp MV (2013) RF-related heating assessment of extracranial neurosurgical implants at $7 \mathrm{~T}$. Magn Reson Imag 31(6): 1029-1034

12. Simmons $A$, Hakansson $K$ (2011) Magnetic resonance safety. In: Magnetic resonance neuroimaging. Humana Press, Totowa, pp $17-28$

13. Heismann B, Ott M, Grodzki D (2015) Sequence-based acoustic noise reduction of clinical MRI scans. Magn Reson Med 73(3): 1104-1109

14. Schaap K (2015) Working with MRI: An investigation of occupational exposure to strong static magnetic fields and associated symptoms. Thesis, Utrecht University. ISBN: 978-90-393-6344-7
15. Patel M, Williamsom RA, Dorevitch S, Buchanan S (2008) Pilot study investigating the effect of the static magnetic field from a 9.4-T MRI on the vestibular system. J Occup Environ Med 50(5): 576-583

16. Atkinson IC, Sonstegaard R, Pliskin NH, Thulborn KR (2010) Vital signs and cognitive function are not affected by 23-sodium and Introduction 19 17-oxygen magnetic resonance imaging of the human brain at 9.4 T. J Magn Reson Imag 32(1): 82-87

17. Chaljub G, Kramer LA, Johnson RF, Johnson RF, Singh H, Crow WN (2001) Projectile cylinder accidents resulting from the presence of ferromagnetic nitrous oxide or oxygen tanks in the MR suite. AJR Am J Roentgenol 177(1): 27-30

18. Nakamura T, Fukuda K, Hayakawa K (2001) Mechanism of burn injury during magnetic resonance imaging (MRI)--simple loops can induce heat injury. Front Med Biol Eng 11(2): 117-129

19. Shellock FG (2000) Radiofrequency energy induced heating during MR procedures: a review. J Magn Reson Imag 12(1): 30-36

20. Shellock FG, Crues JV (2004) MR procedures: Biologic effects, safety, and patient care. Radiology 232(3): 635-652

21. Chakeres DW, Kangarlu A, Boudoulas H, Young DC (2003) Effect of static magnetic field exposure of up to 8 Tesla on sequential human vital sign measurements. J Magn Reson Imag 18 (3): 346-352

22. Capstick M, McRobbie D, Hand J (2008) An investigation into occupational exposure to electro-magnetic fields for personnel working with and around medical magnetic resonance imaging equipment. Report on Project VT/2007/017 of the European Commission Employment, Social Affairs and Equal Opportunities DG. http://www.myesr.org/html/img/pool/VT2007017F inalReportv04.pdf

23. Theysohn JM, Maderwald S, Kraff O, Moenninghoff C, Ladd ME, Ladd SC (2008) Subjective acceptance of 7 Tesla MRI for human imaging. MAGMA 21(1-2): 63-72

24. Piersson AD, Gorleku PN (2017) A national survey of MRI safety practices in Ghana. Heliyon 3(12): e00480

25. ACR Committee on MR Safety: Greenberg TD, Hoff MN, Gilk TB, Jackson EF, Kanal E, McKinney AM, Och JG, Pedrosa I, Rampulla TL, Reeder SB (2020) ACR guidance document on MR safe practices: Updates and critical information 2019. J Magn Reson Imag 51 (2): 331-338

26. Lavdas I, Miquel ME, McRobbie DW, Aboagye EO (2014) Comparison between diffusion-weighted MRI (DW-MRI) at 1.5 and 3 Tesla: A phantom study. J Magn Reson Imag 40(3): 682-690

27. Lavdas I, Behan KC, Papadaki A, McRobbie DW, Aboagye EO (2013) A phantom for diffusion-weighted MRI (DW-MRI). J Magn Reson Imag 38(1): 173-179

28. Chen CC, Wan YL, Wai YY, Liu HL (2004) Quality assurance of clinical MRI scanners using ACR MRI phantom: Preliminary results. J Dig Imag 17 (4): 279-284. https://doi.org/10.1007/ s10278-004-1023-5

29. Price R, Allison J, Clarke G (2015) Magnetic Resonance Imaging Quality Control Manual. ACR, Reston

30. Delakis I, Moore EM, Leach MO, De Wilde JP (2004) Developing a quality control protocol for diffusion imaging on a clinical MRI system. Phys Med Biol 49(8): 1409

Publisher's Note Springer Nature remains neutral with regard to jurisdictional claims in published maps and institutional affiliations. 